\title{
Totally Unimodular Stochastic Programs
}

\author{
Nan Kong \\ Weldon School of Biomedical Engineering, Purdue University \\ 206 S. Martin Jischke Dr., West Lafayette, IN 47907 \\ nkong@purdue.edu \\ Andrew J. Schaefer \\ Department of Industrial Engineering, University of Pittsburgh \\ 1048 Benedum Hall, Pittsburgh, PA 15261 \\ schaefer@ie.pitt.edu \\ Shabbir Ahmed \\ School of Industrial and Systems Engineering, Georgia Institute of Technology \\ 765 Ferst Drive, Atlanta, GA 30332 \\ sahmed@isye.gatech.edu
}

We consider totally unimodular (TU) stochastic programs, that is, two-stage stochastic programs whose extensive-form constraint matrix is TU. We generalize the notion of total unimodularity to apply to sets of matrices and provide properties of such sets. We provide several sufficient conditions on stochastic programs to be TU. When solving TU stochastic problems using the L-shaped method, it is not clear whether the integrality restrictions should be imposed on the master problem. Such restrictions will make each master problem more difficult to solve. On the other hand, solving the linear relaxation of the master typically means sending fractional (and unlikely optimal) solutions to the subproblems, perhaps leading to more iterations. Our computational results investigate this trade-off and provide insight into which strategy is preferable.

Key words: Stochastic Integer Programming; Total Unimodularity; L-shaped Method

\section{Introduction}

Consider the extensive form of a two-stage stochastic mixed-integer program

$$
\min c^{T} x+\sum_{k=1}^{K} p^{k}\left(d^{k}\right)^{T} y^{k}
$$

subject to

$$
\begin{gathered}
T^{k} x+W^{k} y^{k} \geq h^{k}, 1 \leq k \leq K, \\
x \in \mathbb{R}_{+}^{l_{1}} \times \mathbb{Z}_{+}^{n_{1}-l_{1}}, y^{k} \in \mathbb{R}_{+}^{l_{2}} \times \mathbb{Z}_{+}^{n_{2}-l_{2}}, 1 \leq k \leq K .
\end{gathered}
$$

We assume that the underlying uncertainty is modeled with a finite number of scenarios $K$, and each scenario $k, k=1, \ldots, K$, occurs with probability $p^{k}$. The vector $c$ is a known vector in $\mathbb{R}^{n_{1}}$, and for every scenario $k, 1 \leq k \leq K, d^{k}$ is a vector in $\mathbb{R}^{n_{2}}, h^{k}$ is a vector in $\mathbb{R}^{m}, T^{k}$, the technology matrix, is a matrix in $\mathbb{R}^{m \times n_{1}}$, and $W^{k}$, the recourse matrix, is a matrix in $\mathbb{R}^{m \times n_{2}}$. Without loss of generality, we assume that first-stage constraints have been incorporated into each $T^{k}$ matrices, where the corresponding rows are $\mathbf{0}$ in the $W^{k}$ matrices.

The deterministic equivalent of this stochastic program is given by

$$
\min c^{T} x+\mathcal{Q}(x)
$$

subject to

$$
x \in \mathbb{R}_{+}^{l_{1}} \times \mathbb{Z}_{+}^{n_{1}-l_{1}},
$$


where the expected recourse function, $\mathcal{Q}(x)=\sum_{k=1}^{K} p^{k} Q(x, k)$, and

$$
Q(x, k)=\min \left(d^{k}\right)^{T} y
$$

subject to

$$
\begin{gathered}
W^{k} y \geq h^{k}-T^{k} x, 1 \leq k \leq K, \\
y \in \mathbb{R}_{+}^{l_{2}} \times \mathbb{Z}_{+}^{n_{2}-l_{2}} .
\end{gathered}
$$

While $\mathcal{Q}(x)$ is convex if $l_{2}=n_{2}$, i.e., a continuous recourse, with mixed-integer recourse, $\mathcal{Q}$ is in general nonconvex and discontinuous [19], and therefore difficult to solve. Recent exact solution approaches to such problems include Ahmed et al. [1], Kong et al. [9], Ntaimo [12], Sen and Higle [16], Sen and Sherali [17]; and approximation algorithms include Dhamdhere et al. [4], Kong and Schaefer [8], and Shmoys and Swamy [18]. Klein Haneveld and van der Vlerk [7], Schultz [15], and Stougie and van der Vlerk [20] give comprehensive surveys on stochastic integer programming.

Define $B$ to be the constraint matrix of the extensive form of the stochastic program described in $(1)-(3)$,

$$
B=\left(\begin{array}{ccccc}
T^{1} & W^{1} & & & \\
T^{2} & & W^{2} & & \\
\vdots & & & \ddots & \\
T^{K} & & & & W^{K}
\end{array}\right)
$$

Let $M=m \times K$ and $N=n_{1}+n_{2} \times K$, so that $B$ is an $M \times N$ matrix. This paper deals with the cases when such stochastic program can be solved as a stochastic linear program for any integer right-hand side $h$. It is well known that this is true if and only if $B$ is totally unimodular (TU). We call a stochastic program $T U$ if its extensive-form constraint matrix $B$ is TU.

The literature on total unimodularity in deterministic optimization is vast. Nemhauser and Wolsey [11], Padberg [13], and Schrijver [14], provide surveys. Several authors have considered total unimodularity within stochastic programming. Birge and Louveaux [3] describe one sufficient condition for TU stochastic programs and conclude that stochastic programs are unlikely to meet this condition. van der Vlerk [21] provides a class of convex approximations for complete integer recourse models, and shows that when the recourse matrix is TU these approximations are exact. However, there has been no systematic study of total unimodularity in stochastic integer programming.

In this paper, we extend the notion of total unimodularity to a set of matrices, with which we further characterize TU stochastic programs. We also compare two computational options in applying the L-shaped method [22] to solve TU stochastic programs.

The remainder of this paper is organized as follows. In Section 2, we extend the notion of total unimodularity so that it applies to a set of TU matrices. In Section 3, we characterize TU stochastic programs by applying the extended notion of TU to the technology and recourse matrices. We further provide sufficient conditions in two special classes of TU stochastic programs. Section 4 shows the validity of two L-shaped solution strategies in optimizing TU stochastic programs. These two strategies differ in whether the integrality restrictions are imposed on the master problem. We provide computational results in Section 5 that investigate the trade-off between the two strategies, and give conclusions in Section 6 .

\section{An Extension of Total Unimodularity}

Definition 1 An $m \times n$ matrix $A$ is $T U$ if and only if every square submatrix of $A$ has determinant either 0 , 1 or -1 . 
Theorem 1 [5] $A\{0, \pm 1\} m \times n$ matrix $A$ is $T U$ if and only if for every $J \subseteq\{1, \ldots, n\}$, there exists a partition $\left(J^{1}, J^{2}\right)$ of $J$ such that

$$
\left|\sum_{j \in J^{1}} a_{i j}-\sum_{j \in J^{2}} a_{i j}\right| \leq 1 \text { for } i=1, \ldots, m .
$$

We extend the definition of TU to consider a group of matrices.

Definition 2 Let $\mathcal{T}=\left\{A_{1}, \ldots, A_{T}\right\}$ be a set of matrices, where $A_{t} \in \mathbb{R}^{m \times n_{t}}, t=1, \ldots, T$, and let $v \in\{0, \pm 1\}^{m}$. The set $\mathcal{T}$ is $\mathrm{TU}$ with respect to $v$, denoted by $T U(v)$, if for any column subset $J_{t} \subseteq A_{t}, 1 \leq t \leq T$, there exist partitions $\left(J_{t}^{1}, J_{t}^{2}\right)$ such that

$$
\begin{aligned}
& \sum_{j \in J_{t}^{1}} a_{i j}^{t}-\sum_{j \in J_{t}^{2}} a_{i j}^{t} \in\{0,1\} \text { for } v_{i}=-1,1 \leq t \leq T, \\
& \sum_{j \in J_{t}^{1}} a_{i j}^{t}-\sum_{j \in J_{t}^{2}} a_{i j}^{t} \in\{0, \pm 1\} \text { for } v_{i}=0,1 \leq t \leq T,
\end{aligned}
$$

and

$$
\sum_{j \in J_{t}^{1}} a_{i j}^{t}-\sum_{j \in J_{t}^{2}} a_{i j}^{t} \in\{0,-1\} \text { for } v_{i}=1,1 \leq t \leq T .
$$

Definition 2 synchronizes the rows of the matrices in $\mathcal{T}$ by the vector $v$. This extension of total unimodularity will be used in Section 3 to characterize TU stochastic programs. Due to the blockangular structure presented in $B$, the key to the characterization is to identify the relationship between the technology and recourse matrices.

We next provide several properties of a set of $T U(v)$ matrices.

Proposition 1 A matrix $A$ is $T U$ if and only if $\{A\}$ is $T U(v)$ for some $v \in\{0, \pm 1\}^{m}$.

The following proposition shows that two matrices in a set of $T U(v)$ matrices, where $v \in\{ \pm 1\}^{m}$, can be combined into a larger TU matrix.

Proposition 2 If $\left\{A_{1}, \ldots, A_{T}\right\}$ is $T U(v)$ for some $v \in\{ \pm 1\}^{m}$, then the matrix $\left(A_{s} \mid A_{t}\right)$ is $T U$ for all $1 \leq s, t \leq T$.

Proof: Consider any column subset $J=\left(J_{s}, J_{t}\right)$ of $\left(A_{s} \mid A_{t}\right)$. Since $\left\{A_{s}, A_{t}\right\}$ is $T U(v)$, there exist a partition $\left(J_{s}^{1}, J_{s}^{2}\right)$ of $J_{s}$ and a partition $\left(J_{t}^{1}, J_{t}^{2}\right)$ of $J_{t}$ that satisfy the definition of $T U(v)$. The partitions $J^{1}=\left\{J_{s}^{1}, J_{t}^{2}\right\}$ and $J^{2}=\left\{J_{s}^{2}, J_{t}^{1}\right\}$ satisfy Theorem 1.

Proposition $3 A$ set of matrices $\mathcal{T}=\left\{A_{1}, \ldots, A_{T}\right\}$ is $T U(v)$ for all $v \in\{0, \pm 1\}^{m}$ if and only if in each matrix $A_{i}, i=1, \ldots, T$, there is at most one nonzero entry in each column.

Proof: It suffices to check if any $\left\{A_{i}\right\}$ is $T U(v)$ for all $v \in\{0, \pm 1\}^{m}$. The proof of the sufficient condition is trivial. We prove the necessary condition by contradiction. Suppose a column $j$ in a matrix $A_{i}$ has at least two nonzero entries. We arbitrarily select two of the nonzero entries in $j$ and label their rows $s$ and $t$. Let $v_{s}=-a_{s j}$ and $v_{t}=a_{t j}$. If we select column $j$ to be $J$, there does not exist a partition $\left(J_{1}, J_{2}\right)$ that satisfies Theorem 1 .

Remark 1 For any $m \times n(0, \pm 1)$ matrix $A,\{A\}$ is $T U(v)$ for all $v \in\{0, \pm 1\}^{m}$ if and only if $A$ (with duplicate columns removed) is a submatrix of $\left(I_{m},-I_{m}, \mathbf{0}\right)$.

Proposition 4 If $A_{1}$ is $T U$ and contains a column with more than one nonzero entry, then there exists a TU matrix $A_{2}$ such that $\left(A_{1} \mid A_{2}\right)$ is not $T U$. 


\section{Characterizing Totally Unimodular Stochastic Programs}

Consider the two-stage stochastic programming polyhedron defined by

$$
P=\left\{x \in \mathbb{R}_{+}^{n_{1}}, y \in \mathbb{R}_{+}^{n_{2} \times K} \mid T^{k} x+W^{k} y^{k} \geq h^{k}, 1 \leq k \leq K\right\} .
$$

We denote $h=\left(\left(h^{1}\right)^{T},\left(h^{2}\right)^{T}, \ldots,\left(h^{K}\right)^{T}\right)^{T}$.

Theorem 2 [6] The extensive-form constraint matrix $B$ of a two-stage stochastic program is TU if and only if the corresponding polyhedron $P$ is integral for all right-hand sides $h \in \mathbb{Z}^{M}$ for which it is nonempty.

If the extensive-form constraint matrix $B$ is $\mathrm{TU}$, obviously each matrix $T^{1}, \ldots, T^{K}, W^{1}, \ldots, W^{K}$ must be as well, and $B$ must be a $(0, \pm 1)$ matrix. When Theorem 1 is applied to $B$, we obtain the following.

Corollary 1 The extensive-form constraint matrix $B$ of a two-stage stochastic program is $T U$ if and only if for every column subset $J=\left\{J_{0}, J_{1}, \ldots, J_{K}\right\} \subseteq\{1, \ldots, N\}$ there exists a partition $\left(J^{1}, J^{2}\right)=\left\{\left(J_{0}^{1}, J_{1}^{1}, \ldots, J_{K}^{1}\right),\left(J_{0}^{2}, J_{1}^{2}, \ldots, J_{K}^{2}\right)\right\}$ such that

$$
\left|\sum_{j \in J_{0}^{1}} t_{i j}^{k}+\sum_{j \in J_{k}^{1}} w_{i j}^{k}-\sum_{j \in J_{0}^{2}} t_{i j}^{k}-\sum_{j \in J_{k}^{2}} w_{i j}^{k}\right| \leq 1 \text { for } i=1, \ldots, m, k=1, \ldots, K .
$$

Remark $2 B$ is not necessarily $T U$ even if $T^{k}$ and $W^{k}$ matrices for all $k=1, \ldots, K$ are $T U$.

Theorem 3 A two-stage stochastic program is $T U$ if $\left(\left(T^{1}\right)^{T}|\ldots|\left(T^{K}\right)^{T}\right)^{T}$ is $T U$, and each matrix $W^{k}, 1 \leq k \leq K$, is such that $\left\{W^{k}\right\}$ is $T U(v)$ for all $v \in\{0, \pm 1\}^{m}$.

Proof: Since $\left(\left(T^{1}\right)^{T}|\ldots|\left(T^{K}\right)^{T}\right)^{T}$ is TU, for every subset $J \subseteq\left\{1, \ldots, n_{1}\right\}$, there must exist a partition $\left(J^{1}, J^{2}\right)$ of $J$ and a vector $v^{k} \in\{0, \pm 1\}^{m}$ such that

$$
\sum_{j \in J^{1}} t_{i j}^{k}-\sum_{j \in J^{2}} t_{i j}^{k}=v_{i}^{k}
$$

for all $1 \leq i \leq m$ and every $1 \leq k \leq K$. Since each set $\left\{W^{k}\right\}$ is $T U\left(v^{k}\right)$, for each subset of columns of $W^{k}$ there exists a partition such that Corollary 1 is satisfied.

A two-stage stochastic program has simple recourse, if the only recourse action is to incur a linear penalty for shortages or surpluses, that is, the recourse matrix $W^{k}=(I,-I)$ for all $1 \leq k \leq K$.

Corollary 2 A two-stage stochastic program with simple recourse is TU if the matrix $\left(\left(T^{1}\right)^{T}|\ldots|\left(T^{K}\right)^{T}\right)^{T}$ is $T U$.

Remark 3 Simple recourse is the only recourse matrix that admits a TU stochastic program for every TU technology matrix $\left(\left(T^{1}\right)^{T}|\ldots|\left(T^{K}\right)^{T}\right)^{T}$.

Remarks 1 and 3 illustrate the importance of simple recourse in construction of TU stochastic programs. 
Theorem 4 A two-stage stochastic program with fixed technology matrix $T$ is $T U$ if there exists a $v \in\{ \pm 1\}^{m}$ such that $\left\{T, W^{1}, \ldots, W^{K}\right\}$ is $T U(v)$.

Proof: Consider any subset $J=\left\{J_{0}, J_{1}, \ldots, J_{K}\right\}$. Since $\left\{T, W^{1}, \ldots, W^{K}\right\}$ is $T U(v)$, there exists a partition $\left(J_{k}^{1}, J_{k}^{2}\right)$ for each $J_{k}, k=0, \ldots, K$, such that for each $i=1, \ldots, m$,

$$
\begin{gathered}
\sum_{j \in J_{k}^{1}} w_{i j}^{k}-\sum_{j \in J_{k}^{2}} w_{i j}^{k} \in\{0,1\} \text { for } v_{i}=-1,1 \leq k \leq K, \\
\sum_{j \in J_{k}^{1}} w_{i j}^{k}-\sum_{j \in J_{k}^{2}} w_{i j}^{k} \in\{0,-1\} \text { for } v_{i}=1,1 \leq k \leq K, \\
\sum_{j \in J_{0}^{1}} t_{i j}-\sum_{j \in J_{0}^{2}} t_{i j} \in\{0,1\} \text { for } v_{i}=-1,
\end{gathered}
$$

and

$$
\sum_{j \in J_{0}^{1}} t_{i j}-\sum_{j \in J_{0}^{2}} t_{i j} \in\{0,-1\} \text { for } v_{i}=1 .
$$

The partition of $J$ given by $J^{1}=\left\{J_{0}^{2}, J_{1}^{1}, \ldots, J_{K}^{1}\right\}$ and $J^{2}=\left\{J_{0}^{1}, J_{1}^{2}\right.$, $\left.\ldots, J_{K}^{2}\right\}$ satisfies Corollary 1.

We next consider those two-stage stochastic problems where the technology matrix is the identity matrix and the recourse matrix is a special type of interval matrix under each scenario.

Definition 3 [11] An $m \times n(0,1)$ matrix $A$ is called an interval matrix if in each column the 1's appear consecutively; that is, if $a_{i j}=a_{k j}=1$ and $k>i+1$, then $a_{l j}=1$ for all $l$ with $i<l<k$.

In each column $j=1, \ldots, n$, let $\alpha_{j}$ and $\beta_{j}$ index the row of a $(0,1)$ matrix $A$ where the first and last 1 appears, respectively.

Definition 4 An $m \times n$ interval matrix is a nested interval matrix if there exists an ordering of the columns such that $\alpha_{j^{\prime}} \leq \alpha_{j^{\prime \prime}}$ and $\beta_{j^{\prime}} \geq \beta_{j^{\prime \prime}}$, for any $1 \leq j^{\prime}<j^{\prime \prime} \leq n$.

Corollary 3 A two-stage stochastic program is $T U$ if for each $1 \leq k \leq K, T_{k}$ is the identity matrix and $W_{k}$ is a nested interval matrix.

Proof: Consider any column subset $J=\left\{J_{0}, J_{1}, \ldots, J_{K}\right\}$. For each $k=1, \ldots, K$, we assign the $j^{\text {th }}$ column in $J_{k}$ to $J_{k}^{1}$ if $j$ is an odd number and assign the column to $J_{k}^{2}$ otherwise. Hence, by the definition of a nested interval matrix, we have

$$
\sum_{j \in J_{k}^{1}} w_{i j}^{k}-\sum_{j \in J_{k}^{2}} w_{i j}^{k} \in\{0,+1\}
$$

for all $k=1, \ldots, K$. Then the result follows from Corollary 1 by setting $J_{0}^{1}=\emptyset$ and $J_{0}^{2}=J_{0}$. 


\section{Optimizing Totally Unimodular Stochastic Programs}

The L-shaped method [22] applies to TU stochastic programs even with mixed-integer recourse. In this section, we discuss two solution strategies with the L-shaped method. We will first introduce mathematical preliminaries for the two solution strategies. We will then show how to guarantee the two stategies converge to the same optimal solution for TU stochastic programs with some restriction on the solution of the master problem.

Consider the linear relaxation of the recourse problem in the stochastic program described in (1) - (3). Define $Q^{L P}(x, k)$ to be the optimal objective value to the LP relaxation of the recourse problem under scenario $k$ given first-stage solution $x$. Let $\mathcal{Q}^{L P}(x)=\sum_{k=1}^{K} p^{k} Q^{L P}(x, k)$. Clearly, for any $x \in X$ and all scenarios $k, Q^{L P}(x, k) \leq Q(x, k)$ and thus $\mathcal{Q}^{L P}(x) \leq \mathcal{Q}(x)$.

Example 1 Suppose $K=1$ and $T^{1}=W^{1}=I^{m}$, and $Y=\mathbb{Z}_{+}^{m}$. Clearly, the matrix $B$ is $T U$. Note that for any $x, y_{i}^{*}=\left\lceil h_{i}-x_{i}\right\rceil^{+}$for $1 \leq i \leq m$. Therefore, $Q(x, k)=\left(d^{k}\right)^{T}\left(\lceil h-x\rceil^{+}\right)$, and so $\mathcal{Q}(x)$ is nonconvex and discontinuous. Note that $\mathcal{Q}^{L P}(x)=\left(d^{k}\right)^{T}(h-x)^{+}<\mathcal{Q}(x)$ for $h-x \notin \mathbb{Z}_{+}^{m}$.

Example 1 shows that even if $B$ is $\mathrm{TU}$, the expected recourse function $\mathcal{Q}(x)$ is in general nonconvex and discontinuous. Furthermore, there may exist $x \in \mathbb{R}_{+}^{n_{1}}$ such that $\mathcal{Q}^{L P}(x)<\mathcal{Q}(x)$.

Given $x \in \mathbb{Z}_{+}^{n_{1}}$, let $y^{L P}(x, k)$ be an extreme point optimal solution to $Q^{L P}(x, k)$ for $1 \leq k \leq K$, and let $y^{L P}(x)=\left(\left(y^{L P}(x, 1)\right)^{T}, \ldots,\left(y^{L P}(x, K)\right)^{T}\right)^{T} \in \mathbb{R}_{+}^{n_{2} \times K}$. For a TU stochastic program with $h \in \mathbb{Z}^{M}$ and $P$ in (14) is nonempty, let $\left(x^{I P}, y^{L P}\left(x^{I P}\right)\right) \in \mathbb{Z}_{+}^{n_{1}} \times \mathbb{R}_{+}^{n_{2} \times K}$ be an extreme point optimal solution to the TU stochastic program where integrality is relaxed in the second stage. Let $\left(x^{L P}, y^{L P}\right) \in \mathbb{R}_{+}^{n_{1}} \times \mathbb{R}_{+}^{n_{2} \times K}$ be an extreme point optimal solution to the TU stochastic program where integrality is relaxed in both stages. With the above definitions, we have the following results: 1) for any $x \in \mathbb{Z}_{+}^{n_{1}}, \mathcal{Q}(x)=\mathcal{Q}^{L P}(x)$; and 2) both $\left(x^{I P}, y^{L P}\left(x^{I P}\right)\right)$ and $\left(x^{L P}, y^{L P}\right)$ are optimal solutions.

The L-shaped method [22], a variant of the Benders' decomposition [2], adds linear cutting planes to build outer approximations of the recourse function, and solves an iterative master problem that is the first-stage problem plus this approximation. In this section, we consider two solution strategies that differ in the way of solving each master problem. One is to solve the master problem as a linear program and the other is to solve it as an integer program. Additionally, both strategies require that an extreme point optimal solution must be obtained in solving each subproblem. From the above exposition, it is clear that the two solution strategies terminate to the same integer optimal solution if the solution is unique. However, if there are multiple optima, solving the master as a linear program may result in a non-integral solution, as the following example shows.

Example 2 Suppose $K=2$ and $T^{1}=T^{2}=1$ and $W^{1}=W^{2}=[1-1]$. Clearly, the matrix $B$ is TU by Proposition 2. Let $c=0$ and $d^{1}=d^{2}=\left[\begin{array}{ll}1 & 1\end{array}\right]^{T}$. Hence,

$$
Q^{L P}(x, k)= \begin{cases}h^{k}-x & \text { if } x<h^{k}, \\ x-h^{k} & \text { if } x \geq h^{k} .\end{cases}
$$

Suppose $h^{1}=1$ and $h^{2}=2$, each with probability $\frac{1}{2}$. Assume also $0 \leq x \leq 10$.

Because the first-stage objective $c^{T} x=0, \mathcal{Q}(x)$ is also the function $z(x)$ to be minimized (see Figure 1). Suppose we start the L-shaped method at $x^{1}=0$ and solve the master problem as a linear program. We show the sequence of iterations for the L-shaped method as follows.

1. Iteration 1: $x^{1}$ is not optimal, generate the cut $\theta \geq 1.5-x$.

2. Iteration 2 : $x^{2}=10, \theta^{2}=-8.5$ is not optimal, generate the cut $\theta \geq x-1.5$. 
3. Iteration $3: x^{3}=1.5, \theta^{3}=0$ is not optimal, generate the cut $\theta \geq 0.5$.

4. Iteration $4: x^{4}=1.5, \theta^{4}=0.5$, which is optimal, but not integral.

It should be noted that with adding the last L-shaped optimality cut $\theta \geq 0.5$ to the master problem, the optimal solution $x$ is not unique. As a result, solving the master problem as a linear program may not guarantee that the L-shaped method terminates with a feasible optimal solution to the TU stochastic program. However, as Theorem 5 shows, if an extreme point solution is obtained when solving the master problem at the last iteration of the L-shaped method, the first-stage decision must be integral.

Let $P^{M}$ denote the polyhedral set of the feasible solutions $(x, \theta)$ of the master problem when the L-shaped method terminates.

Theorem 5 Suppose the L-shaped method terminates with an optimal solution $(\bar{x}, \bar{\theta})$ that is an extreme point of $P^{M}$. Then if $B$ is $T U, \bar{x} \in \mathbb{Z}_{+}^{n_{1}}$.

Proof: We will prove that the solution $(\bar{x}, \bar{y})$, with $\bar{y}=y^{L P}(\bar{x})$, is an extreme point of $P$, which defines the two-stage stochastic programming polyhedron (14). The result then follows from the total unimodularity of $B$.

Clearly $(\bar{x}, \bar{y}) \in P$. Suppose that $(\bar{x}, \bar{y})$ is not an extreme point of $P$. Then there exists $\left(x^{1}, y^{1}\right)$ and $\left(x^{2}, y^{2}\right)$ in $P$ with $\left(x^{1}, y^{1}\right) \neq\left(x^{2}, y^{2}\right)$ such that $\bar{x}=\frac{1}{2} x^{1}+\frac{1}{2} x^{2}$ and $\bar{y}=\frac{1}{2} y^{1}+\frac{1}{2} y^{2}$.

First, consider the case $x^{1}=x^{2}=\bar{x}$. Then $y^{1}$ and $y^{2}$ (with $y^{1} \neq y^{2}$ ) are feasible solutions to the linear program defining $\mathcal{Q}^{L P}(\bar{x})$ whereas $\bar{y}$ is an extreme point of the linear program defining $\mathcal{Q}^{L P}(\bar{x})$. However, $\bar{y}=\frac{1}{2} y^{1}+\frac{1}{2} y^{2}$, and we have a contradiction.

Now, consider the case $x^{1} \neq x^{2}$. Define $\theta^{1}=\sum_{k=1}^{K} p^{k}\left(d^{k}\right)^{T}\left(y^{k}\right)^{1}$ and $\theta^{2}=\sum_{k=1}^{K} p^{k}\left(d^{k}\right)^{T}\left(y^{k}\right)^{2}$. Note that $\left(y^{i}\right)^{T}=\left(\left(y^{1 i}\right)^{T}, \ldots,\left(y^{K i}\right)^{T}\right)$ for $i=1,2$. Then $\theta^{1} \geq \mathcal{Q}^{L P}\left(x^{1}\right)$ and $\theta^{2} \geq \mathcal{Q}^{L P}\left(x^{2}\right)$, and from the validity of the optimality cuts it follows that $\left(x^{1}, \theta^{1}\right) \in P^{M}$ and $\left(x^{2}, \theta^{2}\right) \in P^{M}$. Moreover, $\frac{1}{2} \theta^{1}+\frac{1}{2} \theta^{2}=\sum_{k=1}^{K} p^{k}\left(d^{k}\right)^{T}\left(\frac{1}{2}\left(y^{k}\right)^{1}+\frac{1}{2}\left(y^{k}\right)^{2}\right)=\sum_{k=1}^{K} p^{k}\left(d^{k}\right)^{T} \bar{y}^{k}=\bar{\theta}$, where the last equality follows from the fact that at termination of the L-shaped method $\bar{\theta}=\mathcal{Q}^{L P}(\bar{x})=\sum_{k=1}^{K} p^{k}\left(d^{k}\right)^{T} \bar{y}^{k}$. We have $\left(x^{1}, \theta^{1}\right)$ and $\left(x^{2}, \theta^{2}\right)$ in $P^{M}$, with $x^{1} \neq x^{2}$ such that $\frac{1}{2}\left(x^{1}, \theta^{1}\right)+\frac{1}{2}\left(x^{2}, \theta^{2}\right)=(\bar{x}, \bar{\theta})$. This contradicts the hypothesis that $(\bar{x}, \bar{\theta})$ is an extreme point of $P^{M}$.

Theorem 5 shows that one only needs to obtain an extreme point solution to each subproblem and obtain an extreme point solution of the last iterative master problem as the L-shaped method terminates. This is normally ensured by using the simplex method for the master problem. The two solution strategies indicate a potential trade-off. When integrality restrictions on the first stage are relaxed, the master problem is easier to solve. However, in this case, fractional solutions $x$ may be generated. Now that there exists an optimal solution with $x$ being integral, solving the original master problem may be advantageous, since every solution to the master problem is potentially an optimal solution. In the next section, we further discuss this tradeoff with our computational experiments.

\section{Computational Experiments Comparing the Two L-shaped Strate- gies}

While certain specialized versions of the L-shaped method may apply to special classes of TU stochastic programs, e.g., those with simple integer recourse [10], the purpose of our experiments 
Table 1: Parameters for generating Classes 1 and 2 instances

\begin{tabular}{c|ccccc|cc}
\hline & \multicolumn{6}{|c|}{ network matrix } & \multicolumn{2}{c}{ SIP } \\
\cline { 2 - 8 } Class & $\begin{array}{c}\text { \# of } \\
\text { nodes }\end{array}$ & $\begin{array}{c}\text { \# of } \\
\text { arcs }\end{array}$ & $\begin{array}{c}\text { \# of } \\
\text { sources }\end{array}$ & $\begin{array}{c}\text { \# of } \\
\text { sinks }\end{array}$ & $\begin{array}{c}\text { Total } \\
\text { supply }\end{array}$ & $\begin{array}{c}\text { 2nd-stage } \\
\text { obj }\end{array}$ & $\begin{array}{c}\text { 2nd-stage } \\
\text { rhs }\end{array}$ \\
\hline 1 & 40 & 65 & 2 & 2 & 30 & $\sim U(5,10)$ & $\sim U\left(0.9 h_{0}, 1.1 h_{0}\right)^{*}$ \\
2 & 70 & 100 & 35 & 35 & 35 & $\sim U(1,10)$ & 1 \\
\hline
\end{tabular}

${ }^{*} h_{0}$ : average first-stage node-wise supply/demand.

Table 2: Parameters for generating Classes 3 and 4 instances

\begin{tabular}{c|cc|cc}
\hline & \multicolumn{2}{|c|}{ nested interval matrix } & \multicolumn{2}{c}{ SIP } \\
\cline { 2 - 5 } Class & $\begin{array}{cccc}\text { \# of } \\
\text { rows }\end{array}$ & $\begin{array}{c}\text { \# of } \\
\text { columns }\end{array}$ & $\begin{array}{c}\text { 1st-stage } \\
\text { obj }\end{array}$ & $\begin{array}{c}\text { 2nd-stage } \\
\text { obj }\end{array}$ \\
\hline 3 & 50 & 90 & $\sim U(1,20)$ & $\sim U\left(0.5 d_{0}, 1.5 d_{0}\right)^{*}$ \\
4 & 80 & 300 & $\sim U(1,5)$ & $\sim U\left(0.5 d_{0}, 1.5 d_{0}\right)^{*}$ \\
\hline
\end{tabular}

is to explore the potential trade-off between the two aforementioned solution strategies. In this section, we call the two strategies, the LP-master strategy and the IP-master strategy, respectively.

We solved two types of TU stochastic programs corresponding to the two sufficient conditions presented in Section 3. The first two problem classes comprise instances in which $T$ is a fixed network matrix and $W$ is $[I,-I]$ for all scenarios. Corollary 2 establishes that these instances are TU. The next two classes comprise instances in which $T$ is identity matrix and $W$ is a fixed nested interval matrix defined in Definition 4. Corollary 3 establishes that these instances are TU.

In each of the 4 classes, we generated 100 TU stochastic programming instances. Tables 1 and 2 report the parameter settings used for the instance generation. In Class 2, we specified that the second-stage objective function coefficients are the only stochastic component for simplicity in the generation. For each instance, we varied the number of scenarios, i.e., $K=1000,2000, \ldots, 10000$ and tested the two solution strategies.

All the instances were solved on a Pentium IV PC with a $2.40 \mathrm{GHz}$ CPU and their computational results are reported in Tables $3-5$. In Table 3, we present the average ratio of the number of cuts required by the IP-master strategy to the number of cuts required by LP-master strategy. In Classes 1 and 2, only optimality cuts are needed since the stochastic integer programs have complete recourse. Regardless of the number of scenarios, solving the IP-master strategy requires fewer cuts on average than the LP master strategy. In Table 4, we present the number of integer incumbent solutions obtained in the L-shaped method with the LP-master strategy, for 10 instances randomly selected from Classes 3 and 4 . These results show that the LP-master strategy converges along a much different path than the IP-master strategy. For some instances with given numbers of scenarios, e.g. Instance 1 from Class 3 with 1000 scenarios, it generates only one integer solution, which is in fact the optimal solution. Since the generated fractional solutions are unlikely to be optimal, these results also indicate that the LP-master strategy tends to require more iterations than the IP-master strategy.

Table 5 reports the percentage of instances in which the IP-master strategy takes less time than the LP-master strategy. In all four classes, the IP-master strategy is more likely to be superior as the number of scenarios increases. This observation can be interpreted as follows. When imposing the integrality restrictions on the master problem, the L-shaped method tends to require fewer iterations, which compensates the extra computational burden caused by solving the IP master. 
Table 3: Average ratio for the number of cuts (LP master vs. IP master)

\begin{tabular}{cccccccccccc}
\hline & & \multicolumn{10}{c}{ Number of Scenarios } \\
\cline { 3 - 12 } Class & Cuts & 1000 & 2000 & 3000 & 4000 & 5000 & 6000 & 7000 & 8000 & 9000 & 10000 \\
\hline 1 & opt. & 1.311 & 1.304 & 1.323 & 1.335 & 1.312 & 1.321 & 1.332 & 1.300 & 1.304 & 1.271 \\
2 & opt. & 1.701 & 1.695 & 1.675 & 1.647 & 1.681 & 1.718 & 1.689 & 1.653 & 1.709 & 1.715 \\
3 & fea. & 1.163 & 1.151 & 1.135 & 1.158 & 1.133 & 1.125 & 1.104 & 1.149 & 1.117 & 1.157 \\
& opt. & 1.068 & 1.149 & 1.099 & 1.082 & 1.067 & 1.079 & 1.111 & 1.122 & 1.082 & 1.112 \\
& total & 1.159 & 1.164 & 1.133 & 1.143 & 1.125 & 1.120 & 1.119 & 1.153 & 1.120 & 1.151 \\
4 & fea. & 1.058 & 1.083 & 1.057 & 1.049 & 1.063 & 1.049 & 1.052 & 1.049 & 1.064 & 1.072 \\
& opt. & 1.541 & 1.543 & 1.517 & 1.495 & 1.387 & 1.529 & 1.506 & 1.515 & 1.508 & 1.536 \\
& total & 1.333 & 1.347 & 1.326 & 1.299 & 1.227 & 1.326 & 1.314 & 1.323 & 1.321 & 1.340 \\
\hline
\end{tabular}

Table 4: Integer incumbent solution in the LP-master strategy (\# of integer incumbent solutions / \# of iterations)

\begin{tabular}{|c|c|c|c|c|c|c|c|c|c|c|}
\hline \multirow[b]{2}{*}{ Class_Inst. } & \multicolumn{10}{|c|}{ Number of Scenarios } \\
\hline & 1000 & 2000 & 3000 & 4000 & 5000 & 6000 & 7000 & 8000 & 9000 & 10000 \\
\hline 3_1 & $1 / 61$ & $4 / 64$ & $5 / 57$ & $2 / 47$ & $2 / 63$ & $1 / 55$ & $1 / 68$ & $1 / 68$ & $1 / 59$ & $2 / 61$ \\
\hline 3_2 & $5 / 52$ & $3 / 65$ & $1 / 62$ & $2 / 70$ & $1 / 56$ & $6 / 60$ & $1 / 47$ & $2 / 54$ & $7 / 67$ & $2 / 60$ \\
\hline 3_3 & $1 / 56$ & $1 / 53$ & $1 / 47$ & $1 / 62$ & $1 / 58$ & $5 / 55$ & $2 / 51$ & $2 / 51$ & $1 / 52$ & $2 / 57$ \\
\hline 3_4 & $1 / 43$ & $1 / 55$ & $1 / 47$ & $1 / 31$ & $2 / 36$ & $1 / 31$ & $6 / 49$ & $1 / 33$ & $4 / 47$ & $1 / 36$ \\
\hline 3_5 & $1 / 41$ & $1 / 57$ & $2 / 42$ & $1 / 56$ & $1 / 55$ & $3 / 51$ & $1 / 51$ & $2 / 56$ & $2 / 54$ & $4 / 59$ \\
\hline 4 4_1 & $3 / 185$ & $5 / 165$ & $4 / 178$ & $7 / 175$ & $2 / 152$ & $4 / 159$ & $4 / 177$ & $5 / 163$ & $5 / 164$ & $6 / 163$ \\
\hline 4_2 & $3 / 154$ & $4 / 155$ & $3 / 162$ & $1 / 160$ & $2 / 140$ & $2 / 163$ & $7 / 169$ & $2 / 160$ & $3 / 153$ & $12 / 165$ \\
\hline 4_3 & $2 / 164$ & $5 / 147$ & $8 / 156$ & $7 / 155$ & $6 / 162$ & $6 / 147$ & $3 / 153$ & $5 / 160$ & $3 / 152$ & $6 / 154$ \\
\hline 4_4 & $1 / 144$ & $6 / 143$ & $3 / 141$ & $5 / 136$ & $5 / 128$ & $2 / 138$ & $1 / 131$ & $2 / 136$ & $5 / 138$ & $1 / 128$ \\
\hline 4_5 & $7 / 119$ & $1 / 121$ & $6 / 117$ & $5 / 121$ & $3 / 120$ & $7 / 127$ & $7 / 114$ & $9 / 132$ & $7 / 115$ & $11 / 132$ \\
\hline
\end{tabular}

\section{Conclusions}

This paper considers two-stage stochastic programs with totally unimodular extensive-form constraint matrices. Such stochastic programs enjoy the integrality property in the sense that every extreme point solution is integral for any integer right-hand sides.

We provide sufficient conditions for a stochastic program to be totally unimodular. Our computational experiments explore under which conditions such stochastic programs should be solved with an integer master or a linear master. Our computational results indicate that when there are relatively few scenarios, solving an integer master is not worth the extra effort. However, as the number of scenarios grows solving fewer master problems becomes more important, and the IP master strategy appears to be more effective.

\section{References}

[1] S. Ahmed, M. Tawarmalani, and N. V. Sahinidis. A finite branch-and-bound algorithm for two-stage stochastic integer programs. Mathematical Programming, 100(2):355-377, 2004.

[2] J. F. Benders. Partitioning procedures for solving mixed-variables programming problems. Numerische Mathematik, 4(1):238-252, 1962.

[3] J. R. Birge and F. V. Louveaux. Introduction to Stochastic Programming. Springer, New York, New York, 1997.

[4] K. Dhamdhere, R. Ravi, and M. Singh. On stochastic minimum spanning trees. In Proceedings of the 11th International Conference on Integer Programming and Combinatorial Optimization (IPCO), pages 321-334, 2005. 
Table 5: Percentage of instances where solving IP masters is faster than solving LP masters

\begin{tabular}{ccccccccccc}
\hline & \multicolumn{10}{c}{ Number of Scenarios } \\
\cline { 2 - 11 } Class & 1000 & 2000 & 3000 & 4000 & 5000 & 6000 & 7000 & 8000 & 9000 & 10000 \\
\hline 1 & 29 & 51 & 59 & 69 & 69 & 73 & 78 & 73 & 75 & 78 \\
2 & 0 & 10 & 17 & 20 & 28 & 33 & 37 & 39 & 47 & 43 \\
3 & 39 & 56 & 58 & 60 & 56 & 58 & 58 & 64 & 62 & 68 \\
4 & 11 & 33 & 45 & 58 & 61 & 72 & 71 & 75 & 79 & 80 \\
\hline
\end{tabular}

[5] A. Ghouila-Houri. Caracterisation des matrices totalement unimodulaires. C. R. Academy of Sciences of Paris, 254:1192-1194, 1962.

[6] A. J. Hoffman and J. B. Kruskal. Integral boundary points of convex polyhedra. In H. W. Kuhn and A. W. Tucker, editors, Linear Inequalities and Related Systems, pages 223-246. Princeton University Press, Princeton, New Jersey, 1956.

[7] W. K. Klein Haneveld and M. H. van der Vlerk. Stochastic integer programming: General models and algorithms. Annals of Operations Research, 85:39-57, 1999.

[8] N. Kong and A. J. Schaefer. A factor $\frac{1}{2}$ approximation algorithm for two-stage stochastic matching problems. European Journal of Operations Research, 172(3):740-746, 2006.

[9] N. Kong, A. J. Schaefer, and B. Hunsaker. Two-stage integer programs with stochastic righthand sides: A superadditive dual approach. Mathematical Programming, 108(2):275-296, 2006.

[10] F. V. Louveaux and M.H. van der Vlerk. Stochastic programming with simple integer recourse. Mathematical Programming, 61(3):301-325, 1993.

[11] G. L. Nemhauser and L. A. Wolsey. Integer and Combinatorial Optimization. Wiley, Hoboken, New Jersey, 1988.

[12] L. Ntaimo. Disjunctive decomposition for two-stage stochastic mixed-binary programs with random recourse, To appear.

[13] M. W. Padberg. Characterizations of totally unimodular, balanced and perfect matrices. In B. Roy, editor, Combinatorial Programming: Methods and Applications, pages 275-284. Reidel, 1975.

[14] A. Schrijver. Theory of Linear and Integer Programming. Wiley, Hoboken, New Jersey, 1986.

[15] R. Schultz. Stochastic programming with integer variables. Mathematical Programming, 97(12):285-309, 2003.

[16] S. Sen and J. L. Higle. The $C^{3}$ theorem and a $D^{2}$ algorithm for large scale stochastic integer programming: Set convexification. Mathematical Programming, 104(1):1-20, 2005.

[17] S. Sen and H. D. Sherali. Decomposition with branch-and-cut approaches for two-stage stochastic mixed-integer programming. Mathematical Programming, 106(2):203-223, 2006.

[18] D. B. Shmoys and C. Swamy. An approximation scheme for stochastic linear programming and its application to stochastic integer programs. Journal of the ACM, 53(6):978-1012, 2006.

[19] L. Stougie. Design and analysis of methods for stochastic integer programming. PhD thesis, University of Amsterdam, 1987. 
[20] L. Stougie and M.H. van der Vlerk. Approximation in stochastic integer programming. In D. Johnson, J. K. Lenstra, and D. Shmoys, editors, Handbook in Operations Research and Management Science, Approximation and Heuristics. Elsevier Science, Amsterdam, The Netherlands, To appear.

[21] M. H. van der Vlerk. Convex approximations for complete integer recourse models. Mathematical Programming, 99(2):297-310, 2004.

[22] R. Van Slyke and R. J-B. Wets. L-shaped linear programs with applications to optimal control and stochastic programming. SIAM Journal on Applied Mathematics, 17(4):638-663, 1969. 


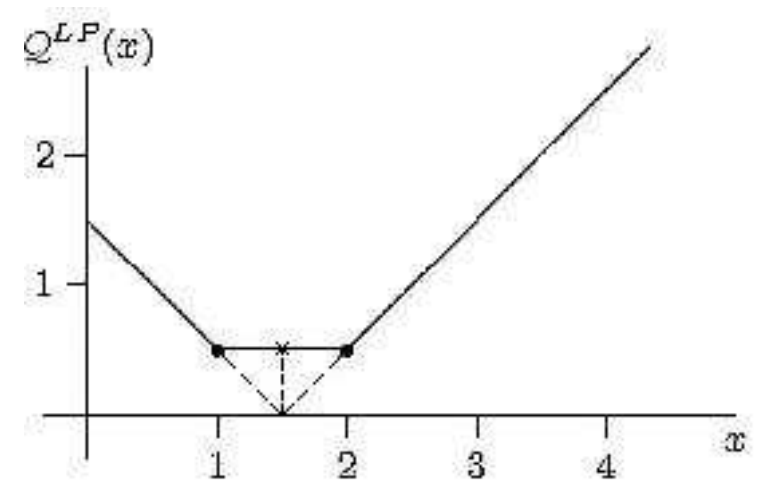

Figure 1: Recourse function for Example 2 